\title{
INFLUENCE OF SECOND-ORDER ELASTICITY ON LOCAL FREDERIKS TRANSITIONS NEAR A NEMATIC LIQUID CRYSTAL-ANISOTROPIC CRYSTAL INTERFACE
}

\author{
H. P. HINOV \\ Institute of Solid State Physics, Bulgarian Academy of Sciences, Sofia 1113, Bulgaria
}

(Reçu le 29 juin 1976, révisé le 25 avril 1977, accepté le 5 mai 1977)

\begin{abstract}
Résumé. - On a étudié théoriquement l'influence de l'élasticité du second ordre $\left(K_{13} \neq 0\right)$ sur la transition locale de Fredericks. Les conditions de seuil sont corrigées considérablement dans les deux transitions fondamentales de l'orientation du cristal liquide nématique homéotrope-conique et conique-planaire. A certaines valeurs de $K_{13} \gtrless 0$, on observe des déformations spontanées.

Abstract. - The influence of second-order elasticity $\left(K_{13} \neq 0\right)$ on local Frederiks transitions is considered theoretically in this paper. The threshold conditions are corrected considerably for the two basic transitions in the alignment of the nematic liquid crystal : homeotropic-conical and conicalplanar. Spontaneous deformations appear for some values of $K_{13} \gtrless 0$.
\end{abstract}

Van der Waals forces play a considerable role in the orientation of the nematic and cholesteric liquid crystals at container interfaces [1-5]. This role is particularly important at a liquid crystal-anisotropic crystal interface. As shown by Smith and Ninham [3], the Van der Waals forces apply a torque to the liquid crystal molecules and align them in the direction of the crystal anisotropic axis. E. Dubois-Violette and P. G. de Gennes [6] considered cases of combined action of the long range Van der Waals forces favouring a homeotropic alignment and the direct steric forces acting close to the interface, bringing about a planar alignment of the liquid crystal. The alignment of the liquid crystal may be homeotropic (with a predominance of the long range component of the Van der Waals forces), planar (with a predominance of the short range steric forces) and intermediateconical alignment depending on the relative proportion of these forces. A similar case was observed by Ryschenkov and Kléman [7] for MBBA at interfaces coated by a thin amorphous layer, with an anisotropic interfacial energy of interaction of the liquid crystal molecules and the amorphous layers $-10^{-4} \mathrm{erg} / \mathrm{cm}^{2}$. De Gennes and Dubois-Violette called transitions between these three basic configurations LOCAL FREDERIKS TRANSITION.

The influence of the second derivatives of the director with respect to the coordinates (second order elasticity) introduced after Oseen [8-10], again by Nehring and Saupe [11], on the local Frederiks transitions is considered theoretically in this paper.
The elastic energy (per $\mathrm{cm}^{2}$ of interface) in this case is determined by the expression :

$$
\begin{aligned}
F & =-\left.(1 / 2) A \sin ^{2} \theta\right|_{z=d}+\left.K_{13} \sin \theta \cos \theta \frac{\mathrm{d} \theta}{\mathrm{d} z}\right|_{z=d}+ \\
& +\int_{d}^{\infty}\left[(1 / 2) U(z) \sin ^{2} \theta+(1 / 2) K\left(\frac{\mathrm{d} \theta}{\mathrm{d} z}\right)^{2}\right] \mathrm{d} z \quad(1)
\end{aligned}
$$

where $A$ is the anisotropic interfacial energy, favouring a planar alignment $(\theta=\pi / 2-\varphi, \varphi \rightarrow 0), U(z)$ gives the Van der Waals torques, bringing about a homeotropic orientation $(\theta \rightarrow 0), K_{13}$ is the second-order elastic coefficient, $K$ is the isotropic elastic coefficient, $d$ is the amorphous layer thickness and $z$ coincides with the anisotropic axis of the crystal.

$U(z)$ contains for an anisotropic crystal a leading component, proportional to $z^{-3}[1-3]$ and is determined following the expression given by Dubois-Violette and de Gennes [6]. We shall assume for simplicity that for $z \rightarrow \infty \quad \mathrm{d} \theta / \mathrm{d} z \rightarrow 0$.

Minimization of (1) with respect to the deformation angle $\theta(z)$ (the interfacial term with $\mathrm{d} \theta / \mathrm{d} z$ is transformed into a term of the inverse function $z(\theta)$ and is varied as one from a functional with moving boundaries) leads to the following Euler-Lagrange equation :

$$
\frac{\mathrm{d}}{\mathrm{d} z} \frac{\mathrm{d} \theta}{\mathrm{d} z}=[U(z) / K] \sin \theta \cos \theta
$$

describing the deformations inside the liquid crystal 
and to the following boundary condition, giving the value of $\mathrm{d} \theta / \mathrm{d} z$ at the interface :

$$
\begin{array}{r}
\left(K-K_{13} \cos 2 \theta\right)\left(\frac{\mathrm{d} \theta}{\mathrm{d} z}\right)^{2}+A \sin \theta \cos \theta\left(\frac{\mathrm{d} \theta}{\mathrm{d} z}\right)- \\
-\left(K_{13} / K\right) U(d) \sin ^{2} \theta \cos ^{2} \theta=\left.0\right|_{z=d}
\end{array}
$$

We shall consider the local stability of the two basic configurations : homeotropic $(\theta=0)$ and planar $(\theta=\pi / 2)$.

It is evident from (1) that the energy of these two configurations is equal when

$$
A=A_{\mathrm{c}}=\int_{d}^{\infty} U(z) \mathrm{d} z .
$$

1. For small values of the anisotropic interfacial energy $(\theta=0)$ the linearized differential eq. (2) :

$$
\frac{\mathrm{d}}{\mathrm{d} z}\left(\frac{\mathrm{d} \theta}{\mathrm{d} z}\right)=(U(z) / K) \theta
$$

has a solution $\theta(z)$ which is a decreasing function of $z$ [6] $(\theta(z)<\theta(d))$.

Following Dubois-Violette and de Gennes [6], the torque at the interface can be transformed into :

$$
-\left.K\left(\frac{\mathrm{d} \theta}{\mathrm{d} z}\right)\right|_{z=d}=\int_{d}^{\infty} U(z) \theta(z) \mathrm{d} z .
$$

The threshold condition for a homeotropic configuration is obtained from (6) and (3) :

$$
\begin{aligned}
A^{\prime}=(K- & \left.K_{13}\right) \int_{d}^{\infty} \frac{U(z)}{K} \frac{\theta(z)}{\theta(d)} \mathrm{d} z- \\
& -\frac{K_{13}}{K} U(d)\left[\int_{d}^{\infty} \frac{U(z)}{K} \frac{\theta(z)}{\theta(d)}\right]^{-1} \mathrm{~d} z .
\end{aligned}
$$

2. For large values of the anisotropic interfacial energy $(\theta=\pi / 2)$ the local stability is investigated under the condition that $\varphi=(\pi / 2)-\theta$ is a small angle.

The linearized equation for $\varphi(z)$

$$
\frac{\mathrm{d}}{\mathrm{d} z} \frac{\mathrm{d} \varphi}{\mathrm{d} z}=-(U(z) / K) \varphi
$$

has a solution $\varphi(z)$ which is an increasing function of $z(\varphi(z)>\varphi(d))$.

The local threshold is determined in a similar way by the expression

$$
\begin{aligned}
A^{\prime \prime}=(K+ & \left.K_{13}\right) \int_{d}^{\infty} \frac{U(z)}{K} \frac{\varphi(z)}{\varphi(d)} \mathrm{d} z- \\
& -\frac{K_{13}}{K} U(d) \int_{d}^{\infty}\left[\frac{U(z)}{K} \frac{\varphi(z)}{\varphi(d)}\right]^{-1} \mathrm{~d} z .
\end{aligned}
$$

It is evident that an intermediate conical alignment is realized when $A^{\prime}<A<A^{\prime \prime}$. The expressions (7) and (9) are calculated for the case of an anisotropic crystal when $U(z) / K=B / z^{3}$ under the condition that the thin amorphous layer has a thickness $d$ smaller than $1000 \AA$. Otherwise, the dependence of the Van der Waals torques on the distance $z$ must follow the $z^{-4}$ law (a case considered by Dubois-Violette and de Gennes [5] because of the introduction of the retardation effects in the Van der Waals interactions).

A. For small values of the anisotropic interfacial energy $(\theta=0)$ the solution of $(5)$ in accordance with [6] is expressed with the aid of modified Bessel functions $I_{0}$ and $I_{1}$ :

$$
\theta=\theta(d)(z / d)^{1 / 2} \frac{I_{1}(t)}{I_{1}\left(t_{0}\right)}
$$

where

$$
t=2(B / z)^{1 / 2}, \quad t_{0}=2(B / d)^{1 / 2} .
$$

The substitution of (10) in (7) gives the final expression for the threshold above which the homeotropic configuration is not stable $\left(K_{13}<0\right)$ :

$$
\begin{aligned}
\frac{A^{\prime} B}{K}=\frac{K+\left|K_{13}\right|}{K}(B / d)^{3 / 2} \frac{I_{2}\left[2(B / d)^{1 / 2}\right]}{I_{1}\left[2(B / d)^{1 / 2}\right]}+ \\
+\left(\left|K_{13}\right| / K\right)(B / d)^{3 / 2} \frac{I_{1}\left[2(B / d)^{1 / 2}\right]}{I_{2}\left[2(B / d)^{1 / 2}\right]}
\end{aligned}
$$

B. For large values of the anisotropic interfacial energy $(\theta=\pi / 2)$ the solution of (8) according to [6] is expressed in terms of normal Bessel functions $J_{0}$ and $J_{1}$ :

$$
\varphi=\varphi(d)(z / d)^{1 / 2} \frac{J_{1}(t)}{J_{1}\left(t_{0}\right)}
$$

The ratio $\varphi(z) / \varphi(d)$ is positive for all physical values of $d$ when $d>d_{\mathrm{c}}=0.27 \mathrm{~B}$.

The substitution of (12) in (9) gives the final expression of the threshold over which the planar configuration is not stable $\left(K_{13}<0\right)$ :

$$
\begin{array}{r}
\frac{A^{\prime \prime} B}{K}=\frac{K-\left|K_{13}\right|}{K}(B / d)^{3 / 2} \frac{J_{2}\left[2(B / d)^{1 / 2}\right]}{J_{1}\left[2(B / d)^{1 / 2}\right]}+ \\
+\left(\left|K_{13}\right| / K\right)(B / d)^{3 / 2} \frac{J_{1}\left[2(B / d)^{1 / 2}\right]}{J_{2}\left[2(B / d)^{1 / 2}\right]}
\end{array}
$$

$A^{\prime \prime}$ is diverging when $d \rightarrow d_{\mathrm{c}}$. The solution of eq. (12) for $d<d_{\mathrm{c}}$ is oscillating and does not represent a stable state of the liquid crystal.

The numerical calculation of (11) and (13) (Fig. 1) shows that for $d>d_{\mathrm{c}}$ two transitions exist : for $A=A^{\prime}$ and for $A^{\prime}=A^{\prime \prime}$.

The influence of second-order elasticity $\left(K_{13} \neq 0\right)$ is considerable and corrects these transitions substantially. 


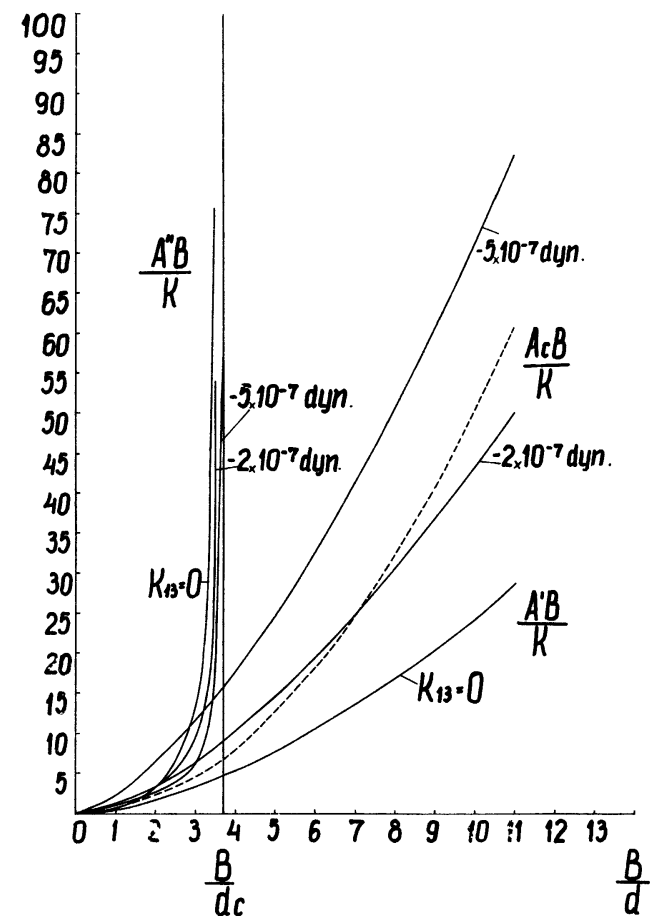

Fig. 1. - Reduced anchoring energy thresholds for $K_{13}<0$

$$
\frac{A_{\mathrm{c}} B}{K}, \quad \frac{A^{\prime} B}{K}, \quad \frac{A^{\prime \prime} B}{K}
$$

A negative value of $K_{13}$ for a fixed $B / d$ decreases $A^{\prime \prime}$ and increases $A^{\prime}$ showing that the interfacial energy, determined by the second derivatives of the director with respect to the coordinates, acts together with the

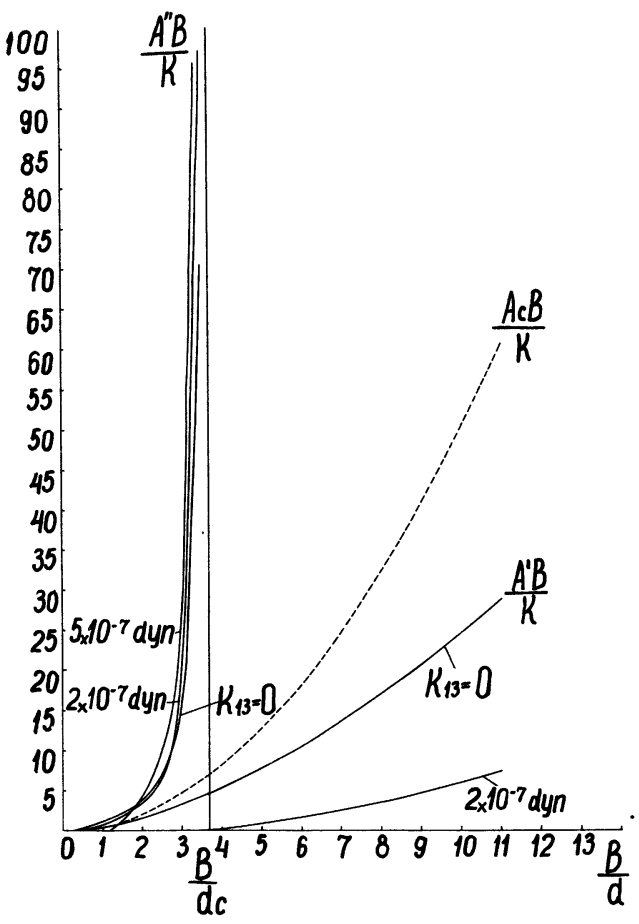

FIG. 2. - Reduced anchoring energy thresholds for $K_{13}>0$

$$
\frac{A_{\mathrm{c}} B}{K}, \quad \frac{A^{\prime} B}{K}, \quad \frac{A^{\prime \prime} B}{K} .
$$

anisotropic Van der Waals energy, causing a homeotropic alignment and at the same time hindering the planar configurations.

A positive value of $K_{13}$ (far less probable as demonstrated theoretically by Nehring and Saupe [12]) exerts a diverse influence on the thresholds $A^{\prime}$ and $A^{\prime \prime}$ (Fig. 2). The large value of the elastic coefficient $K_{13}$ brings $A^{\prime}$ to zero for a certain value of $B / d$ after which $A^{\prime}$ becomes negative. This in our opinion corresponds to spontaneous deformation at $A^{\prime}>0$. For $K_{13}$ of the order of $5 \times 10^{-7}$ dyne, deformation is possible only for $A^{\prime}<0$ (spontaneous deformations $A^{\prime}>0$ ). Smaller values of $K_{13}$ sharply decrease $A^{\prime}$ and have a diverse influence on $A^{\prime \prime}$ depending on the value of $K_{13}$ (Fig. 2).

The order of the local Frederiks transition may be determined from the expression (3) where $\sin \theta$ and $\cos \theta$ are expanded to third-order in $\theta$ and from (6) The following relation is obtained from (3) and (6) for $\theta(d)$ a little over the threshold :

$$
\theta^{2}(d)=(3 / 2) \frac{\Delta A}{A_{K_{13}=0}^{\prime}+\Delta A}
$$

where

$$
\begin{aligned}
& A_{K_{13}=0}^{\prime}=(K / B)(B / d)^{3 / 2} \frac{I_{2}\left[2(B / d)^{1 / 2}\right]}{I_{1}\left[2(B / d)^{1 / 2}\right]} \\
& A_{K_{13}=0}+\Delta A=\frac{A^{\prime}+\Delta A^{\prime}}{2\left(K+K_{13}\right)}+ \\
&+ {\left[\left(\frac{A^{\prime}+\Delta A^{\prime}}{2\left(K+K_{13}\right)}\right)^{2}-\frac{K_{13} U(d)}{K\left(K+K_{13}\right)}\right]^{1 / 2} } \\
& K_{13} \cos 2 \theta \sim K_{13}, \quad \theta(d) \neq 0 .
\end{aligned}
$$

When $\Delta A \rightarrow 0, \theta(d) \rightarrow 0$, which determines a local Frederiks transition of the second order. In a similar way it is shown that the other transitions for $K_{13} \lessgtr 0$ are of the second order.

Coǹcluding remarks. -1 . THE APPROXIMATION :

$K_{11}^{\prime}=K_{11}-2 K_{13}=K_{33}^{\prime}=K_{33}+2 K_{13}=K$

is used in the energy functional (1) where $K_{11}^{\prime}, K_{33}^{\prime}$ and $K_{13}$ are the elastic coefficients of splay, bend and second-order splay-bend respectively introduced by Nehring and Saupe [11] and $K_{11}$ and $K_{33}$ are the elastic coefficients of splay and bend respectively introduced by Frank [13]. The basic inequalities for the elastic coefficients in Frank's theory were obtained theoretically by Ericksen [14]. It seems to be impossible at the present moment to obtain in a unified manner the inequalities for the elastic coefficients in the theory of Nehring and Saupe. They can be obtained however from the theoretical consideration of partial cases of deformation of a free nematic layer [15]. The authors 
obtained the following relations between $K_{13}$ and $K_{11}^{\prime}$ and $K_{\mathbf{3} 3}^{\prime}$ :

$$
K_{11}^{\prime}-\left|K_{13}\right|>0, \quad K_{33}^{\prime}-\left|K_{13}\right|>0
$$

which are based on the fact that the nematic liquid crystal in its elastic energy minimum is not deformed.

It is evident that Frank's coefficients

$$
K_{11}=K_{11}^{\prime}+2 K_{13} \text { and } K_{33}=K_{33}^{\prime}-2 K_{13}
$$

may be negative as well if $K_{13}<0$ or respectively $K_{13}>0$ and $2\left|K_{13}\right|>K_{11}, K_{33}$. This was considered by Nehring and Saupe [12] in their theoretical paper on the calculation of the elastic coefficients based on a simple internal field model. The following relations between the elastic coefficients included in their model were obtained :

$K_{11}^{\prime}: K_{22}: K_{33}^{\prime}: K_{24}: K_{13}=5: 11: 5:-9:-6$.

These ratios are rather approximate and evidently do not correspond to the derived relations between $K_{11}^{\prime}, K_{33}^{\prime}$ and $K_{13}$. It is evident from all that is stated above that in (15) the isotropic elastic modulus $K$ should be larger than $K_{13}$.

2. IT IS CLEAR FROM FigURE 2 THAT FOR $K_{13}>0$ THE RATIO $K_{13} / K$ MAY BE OBTAINED FROM THE INTERSECTION OF THE CURVE $\frac{A^{\prime} B}{K_{1}}\left(\frac{B}{d}\right)$ WITH THE ABSCISSA :

$$
\frac{K_{13}}{K}=\left[1+\frac{I_{1}^{2}\left(2(B / d)^{1 / 2}\right)}{I_{2}^{2}\left(2(B / d)^{1 / 2}\right)}\right] .
$$

In a physical sense this means that when $2(B / d)^{1 / 2}$ is properly chosen depending on the eventual value of $K_{13} / K$ it is possible to proceed from a stable homeotropic layer (for small values of the surface energy causing vertical orientation : $10^{-3}-10^{-4} \mathrm{erg} / \mathrm{cm}^{2}$ ) to conical configuration.

In a similar manner for $K_{13}<0, K_{13} / K$ may be determined from the same relation for a case where the anisotropic axis of the crystal is oriented along $x$ and the surface energy determines a homeotropic configuration (a transition from a stable homeotropic layer with large surface energy of interaction : $10^{-1}-10^{-2} \mathrm{erg} / \mathrm{cm}^{2}$ to a conical one). The thresholds (11) and (13) then have the respective forms :

$$
\begin{array}{r}
\frac{A^{\prime} B}{K}=\frac{K+\left|K_{13}\right|}{K}(B / d)^{3 / 2} \frac{J_{2}\left[2(B / d)^{1 / 2}\right]}{J_{1}\left[2(B / d)^{1 / 2}\right]}- \\
-\frac{\left|K_{13}\right|}{K}(B / d)^{3 / 2} \frac{J_{1}\left[2(B / d)^{1 / 2}\right]}{J_{2}\left[2(B / d)^{1 / 2}\right]} \\
\frac{A^{\prime \prime} B}{K}=\frac{K-\left|K_{13}\right|}{K}(B / d)^{3 / 2} \frac{I_{2}\left[2(B / d)^{1 / 2}\right]}{I_{1}\left[2(B / d)^{1 / 2}\right]}- \\
-\frac{\left|K_{13}\right|}{K}(B / d)^{3 / 2} \frac{I_{1}\left[2(B / d)^{1 / 2}\right]}{I_{2}\left[2(B / d)^{1 / 2}\right]}
\end{array}
$$

where $d$ is the thickness of a surfactant causing a homeotropic orientation of the liquid crystal.

The thresholds obtained theoretically may be observed in an experiment with a finely ground crystal (with roughness of the order of $10 \AA$ ) and amorphous film (for $K_{13}>0$ ) or a surfactant (for $K_{13}<0$ ). The changes in the orientation of the liquid crystal close to the interface anisotropic crystal-liquid crystal may be investigated with the aid of a total internal reflection of a laser beam [16] with penetration thickness of the damped wave from 500 to $6000 \AA$. The experiment may be changed so that one goes from a local Frederiks transition to a transition in the entire cell when the problem is symmetrized. Depending on the distance between the two surfaces with a liquid crystal between them, one can use the theory of Dubois-Violette and de Gennes [5, 6], or an exact solution of the problem can be obtained on the basis of the surface modes $[2,3$, $17,18]$

3. THE INFLUENCE OF THE TYPE OF SURFACE ENERGY on the results obtained is of interest (refer to DuboisViolette and de Gennes [6]). We will consider the surface energy with a sharp minimum around a certain deformation angle (we will assume zero for simplicity) while constant elsewhere.

We consider the following mathematical form as most convenient for representing such energy :

$$
W_{\mathrm{s}}= \begin{cases}A \sin ^{2} n \varphi & n \varphi \leqslant \pi / 2 \\ A & n \varphi>\pi / 2 .\end{cases}
$$

In the general case the coefficient $A$ is an unknown function of $n$. Formula (21) shows that the sharper minimum around zero, leads to a steeper change in $\varphi$. The application of this function to the problem considered shows that $A^{\prime \prime} B / K$ should be replaced with $A^{\prime \prime} n^{2} B / K$ for a minimum around $\varphi$ zero. The liquid crystal is stable around $\varphi=\pi / 2$ for rather weak Van der Waals forces as well $\left(W_{\mathrm{s}}=A\right)$. For $K_{13}>0$, the liquid crystal is stable around $\varphi=\pi / 2$ up to values of $B / d$ where

$$
\begin{aligned}
& \frac{K-\left|K_{13}\right|}{K} \frac{I_{2}\left[2(B / d)^{1 / 2}\right]}{I_{1}\left[2(B / d)^{1 / 2}\right]}- \\
& \quad-\frac{\left|K_{13}\right|}{K} \frac{I_{1}\left[2(B / d)^{1 / 2}\right]}{I_{2}\left[2(B / d)^{1 / 2}\right]} \geqslant 0
\end{aligned}
$$

The case where the surface energy is at a minimum around $\theta=0$ is considered in a similar manner. The threshold behaviour of the problem considered is eliminated at any other angle $\theta, \varphi \neq 0, \pi / 2$. It is evident from eq. (3), (6) and (14) altered in accordance with (21) that the local Frederiks transition is of the second order.

The preliminary estimates show that the surface energy with a very sharp minimum (e.g. of the type $W_{\mathrm{s}} \sin ^{4} n \theta$ at $n \theta \leqslant \pi / 2$ and $W_{\mathrm{s}}$ at $n \theta>\pi / 2$ ) determines 
a local as well as a general Frederiks transition of the first order [19].

The above theoretical results show that the local Frederiks transition is very convenient for determining the sign and order of $K_{13}$ which is desirable in order to clarify the degree of influence of second-order elasticity on the various static deformations in nematic and cholesteric liquid crystals for small values of the anisotropic interfacial energy.

The author expresses his gratitude to Dr. A. Derzhanski and Dr. A. G. Petrov for the helpful discussions.

\section{References}

[1] De Gennes, P. G., C. R. Hebd. Séan. Acad. Sci. 271 (1970) 469.

[2] Richmond, P., White, L. R., Mol. Cryst. Liq. Cryst. 27 (1974) 217.

[3] Smith, E. R., Ninham, B. W., Physica 66 (1973) 111

[4] KATs, E. I., Zh. Eksp. Theor. Fiz. 70 (1976) 1394.

[5] Dubois-Violette, E., De Gennes, P. G., J. Colloid Int. Sci. 57 (1976) 403.

[6] Dubois-Violette, E., De Gennes, P. G., J. Physique Lett. 36 (1975) L-255.

[7] Ryschenkow, G., Kléman, M., J. Chem. Phys. 64 (1976) 404.

[8] Oseen, C. W., Ark. Mat. Astron. Fys. A 19 (1925) 1.

[9] Oseen, C. W., Fortschr. Chem. Phys. Phys. Chem. 20 (1929) 1.

[10] OseEn, C. W., Trans. Faraday Soc. 29 (1933) 883.
[11] Nehring, J., SAuPe, A., J. Chem. Phys. 54 (1971) 337.

[12] Nehring, J., Saupe, A., J. Chem. Phys. 56 (1972) 5527.

[13] Frank, F. C., Discuss. Faraday Soc. 25 (1958) 19.

[14] Ericksen, J. L., Phys. Fluids 9 (1966) 1205.

[15] Hinov, H. P., Derzhanski, A. I., to be published.

[16] Hinov, H. P., Sainov, S., VI Nat. Conf. on Spect., Slanchev Bryag, Bulgaria Sept. 30-Oct. 3 (1974) Abstracts 248 ; to be published in a revised form.

[17] Van Kampen, N. G., Nuboer, B. R. A., Schram, K., Phys. Lett. 26A (1968) 307.

[18] Richmond, P., Ninham, B. W., J. Phys. C 4 (1971) 1988

[19] Hinov, H. P., DerzhansKi, A. I., to be published. 